\title{
Developing a technology enabled workflow to aid space layout communication for students with Autism Spectrum Disorder - A project case study
}

\author{
Josephine McGoldrick ${ }^{1}$, David Comiskey ${ }^{1}$, Shauna Donnelly ${ }^{2}$ \\ ${ }^{1}$ Belfast School of Architecture and the Built Environment, Ulster University, Northern \\ Ireland, ${ }^{2}$ Positive Behaviour Specialist, Northern Ireland.
}

\begin{abstract}
Within the higher education sector there has been a shift towards flexible teaching and learning spaces that can be re-imagined and re-organised depending on the teaching approach to be employed. While this has undoubted benefits in terms of student engagement, innovative delivery, and exciting learning approaches, it potentially presents a challenge for a student group, those with a diagnosis of Autism Spectrum Disorder (ASD), that has not been fully considered to this point. This study draws upon advances in the Architectural Technology discipline to develop a methodology which can assist in designing and communicating proposed teaching environments, with a particular focus on the potential to benefit students with ASD. The methodology is presented in the form of a case study. The aim is to assist designers and end users in analysing and defining the necessary links to evaluate solutions for the layout planning of spaces, determining functionality and whether proposals are feasible. The output obtained from this workflow elevates its potential in aiding space layout planning, helping those who need assistance in understanding newly formed layouts.
\end{abstract}

Keywords: Learning environment; technology; space layout planning; ASD. 


\section{Introduction}

From an architectural perspective, space-layout planning is a critical part of successful design development. Guo \& Li (2017) define layout design as determining "the shapes, dimensions, and positions of internal building spaces to satisfy architectural criteria." One of the most critical building typologies in respect of space-layout planning is educational, as learning spaces have been said to reflect an institution's teaching and learning "philosophy" and potentially impact on learning and teaching styles (Oblinger, 2005). Within the higher education sector there has been a shift towards flexible teaching and learning spaces, purposely designed for swift layout change depending on the teaching method employed. Some excellent literature on both learning space design and classroom layout planning exists, but the extent to which this guidance is implemented in practice by academics prior to teaching sessions remains unclear. For example, many will meticulously plan the pedagogical approach to be employed for a particular teaching session, but, based on experience, it is questionable as to whether the layout of the space will have been given equal consideration. Yes, academics may have a general idea of how they may want to arrange the space, in terms of desk and seating positions, to align with their pedagogical approach. However, the approach to layout planning is often quite limited, and for good reason, due to unfamiliarity with the environment, time pressures and the physical exertion required.

With it being suggested that space planning requires the enhancement of current methodologies, including involvement of the end user during the process of design (Ciolfi, 2004; Scott, 2009), there is the potential to develop a novel approach to facilitate such a workflow for educational spaces. This could involve end users, in this instance academics and students, as co-creators in the planning of teaching spaces. Such an approach would allow for consideration of the specific needs of students in a particular cohort, for example, those presenting with diagnosed intellectual disabilities. Whilst flexible teaching and learning environments have many benefits, such as encouraging collaboration and personalised learning, they also have the potential to prove challenging for students diagnosed with Autism Spectrum Disorder (ASD).

In Northern Ireland in 2019/20, children with an autism diagnosis made up approximately $4.2 \%$ of the school aged population (Rodgers \& McCluney, 2020). Such data is of importance to education providers in terms of helping inform space layout planning and pedagogical approaches to teaching delivery as these children progress to the next stage of their educational journey. Individuals with ASD tend to prefer routine, and continuous layout changes can negatively impact their learning experience and mental state. This assertion is supported by the content of a 2018 report by Stackhouse (2018) entitled The Impact of 'Place' on Autistic Children. As such, there needs to be a balance between the needs of the individual, the teaching approach employed, and the layout of spaces. So how can this be achieved? It has been suggested that individuals with ASD have a natural allure for technology (Valencia 
et al. 2019), this is where the link with the Architectural Technology (AT) discipline emerges. Due to the use of technology within the built environment sector increasing over recent years, many of those designing learning environments already posess the skillset to harness new design and communication methods and apply them in novel areas. With this work we aim to develop a methodology which can assist in designing and communicating proposed teaching environments, with a particular focus on the potential to benefit individuals diagnosed with ASD. It has been acknowledged that there is a lack of research relating to the broad area of place and ASD (Stackhouse, 2018 citing Vazquez \& Torres, 2013), with this work we hope to contribute to closing the knowledge gap. It should be noted that the focus of this paper is on the layout of the physical environment once constructed and how this is communicated to end users.

\section{Autism Spectrum Disorder, Flexible Learning Spaces \& Technology}

ASD is a developmental disorder that affects how a person can communicate, interact and behave in certain situations. According to the NHS (2019), individuals on the autism spectrum may "get anxious or upset about unfamiliar situations and social events". As such, it is reasonable to assume that flexible learning and teaching environments with constantly changing layouts could have a negative impact on the student experience. This view is strengthened by Vazquez \& Torres (2013), cited by Stackhouse (2018), who suggest that moving spaces and changing activity can be sufficient to trigger a panic attack in an individual with ASD due to "the inability to 'imagine' and create a mental image of what might be on the other side of the door...”. McAllister \& Hadjri (2013), cited by Stackhouse (2018), use the term 'frightening' when describing some pupils perception of educational environments. Stackhouse (2018), citing the work of others, provides some perspective by cautioning that there needs to be a balance between catering for the needs of pupils or students and preparing them for the wider world environment.

\subsection{Learning and Teaching Environments}

It is broadly accepted that teachers should accommodate their students' diverse needs by employing teaching methods which resonate with students of all ability levels (Zeppetella, 2009). However this does not always occur, perhaps due to a lack of understanding of what constitutes good practice. Zeppetella (2009) identified ten practices to help students with Asperger's Syndrome (which falls under ASD) in the classroom. The findings suggested that teachers mostly employ a 'visual' strategy. This approach, combined with strategic positioning within the classroom (carefully considering location, lighting, noise, and ventilation) is important in creating a comfortable learning environment. Such an approach is more achievable nowadays as learning spaces no longer solely consist of fixed layout lecture theatres (Comiskey et al. 2016). It is also important to acknowledge the impact that 
the COVID-19 pandemic may have in respect of this wider discussion. The prolonged period of near exclusive remote delivery brought about by the pandemic has most likely fast tracked the move towards increased adoption of blended learning approaches. It has also seen the emergence of the hybrid classroom concept, that being a combination of students physically present in the learning environment with others joining virtually. Both flexible learning environments and hybrid classrooms in which layouts constantly change, whilst having notable benefits, have the potential to inadvertently increase anxiety levels with ASD students as changes to 'the normal' can cause setbacks in how they function through everyday life. As such, research into methods to reduce stress and anxiety must be advanced.

\subsection{Use of Technology}

As has already been alluded to, an area with potential for reducing stress and anxiety for ASD students is the use of technology, both for partaking in layout design exercises and communicating space layouts in advance of teaching sessions. A report by Lui et al. (2017) concluded that Augmented Reality (AR), via smartglass use, "may have an important future role in helping address the therapeutic needs of children with ASD". Individuals on the autism spectrum appear to respond well to AR technologies as they react powerfully to imagery and graphical content. This is where the link between approaches used within the AT discipline and communication with ASD students becomes apparent. By their very nature, architectural design approaches and outputs are visual due to the need to communicate design concepts to a wide audience. Recently, increased use of technology within the discipline, a combination of government mandate and increased focus on building performance, has exposed more practices to the benefits of technology. This has instigated further exploration, such as the use of laser scanning for data capture, and augmented and virtual reality applications for communication. Augmented reality "adds context by overlaying data and other information on the real world for the end user" (Sargent, 2019). Meanwhile, Virtual Reality (VR) simulates "real world situations and scenarios" in a computer-generated three-dimensional environment, immersing the user in their surroundings (Sargent, 2019). Such advances have provided an opportunity to harness the power of these technologies and use them in novel ways such as outlined in the case study which follows.

\section{Research Methodology}

This study could be described as real world research (Robson \& McCartan, 2016), utilising a qualitative research methodology in the form of a case study to generate an in-depth, multifaceted understanding of the potential for technology to aid the design and communication of flexible teaching space layouts. A case study approach was selected as it allows for indepth exploration of a 'case' and retains a holistic and real-world perspective (Yin, 2018). 
The definition of the methodology follows the steps depicted in Figure 1. It is based on the potential to use technology related advances in design disciplines and adapt them to the aim of this study. After selecting a suitable teaching space, the first stage involved identifying a way of capturing the existing layout (Step 1) and bringing this into an environment where it could be modelled in three-dimensions to create a virtual representation of the space (Step 2). This facilitated space layout exploration (Step 3) before integration with virtual and augmented reality communications platforms was investigated (Step 4). The following case study explains the workflow process in greater detail.

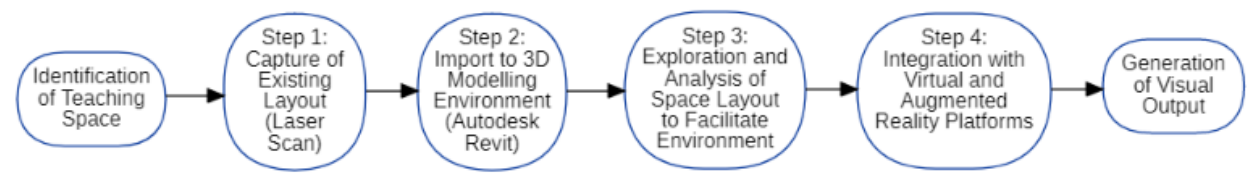

Figure 1. Technology Enabled Workflow.

\section{Case Study}

The flexible learning space identified for the case study was a design studio located on the Jordanstown Campus of Ulster University (Figure 2). Step 1 involved capturing the environment for the purpose of creating a virtual three dimensional model of the space. The use of laser scanning technology was deemed most appropriate for data capture due to its accuracy, image generation, and ability to be processed and imported into architectural design software, in this case Autodesk Revit, to construct an accurate model of the space. It should be noted that the data capture for Step 1 and a basic model for Step 2 had been captured and created by Leica Geosystems prior to this study and was made available for use in this paper.

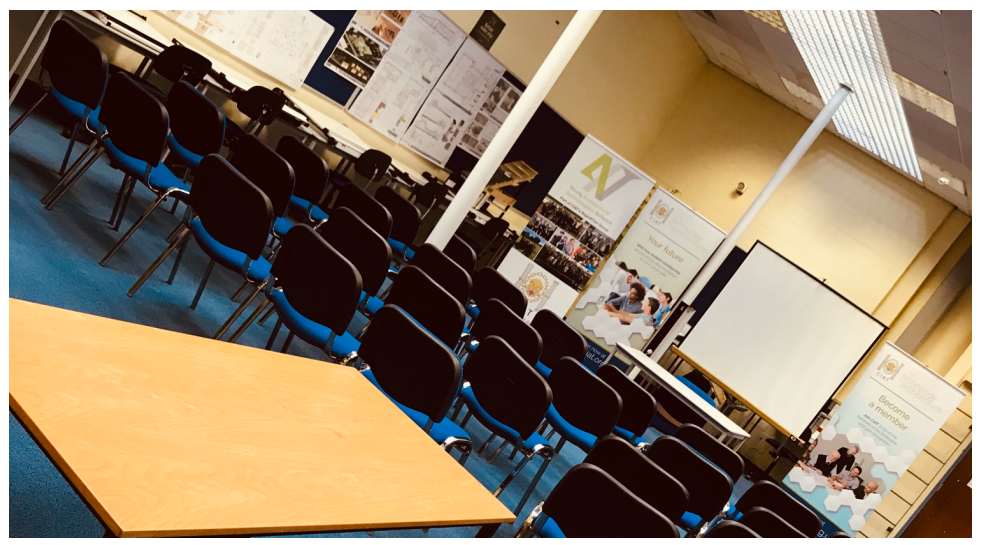

Figure 2. Flexible learning space used in the study. 
Autodesk Revit allows for the use of bespoke furtiture elements, meaning an exact replica of the real world environment could be created from which layouts were optimised. Whilst advances in technology have seen the potential for automated layouts to be generated, this study used a manual approach for the design of a hypothetical teaching space layout. The layout generated was purposely simplistic, influenced by a review of literature and professional experience, and took into account the possibility for optimum positioning for students with Specific Learning Difficulties (SpLDs) especially ASD. The literature identified that students prefer a space that provides minimal distractions (Holtz et al., 2004) while having accessibility to their tutor for ease of communication, as those with ASD can struggle with verbal correspondence. Ganz (2007) and Jansen et al. (2016) determined that visual organisation and environmental components should be contemplated when choosing consistent accommodations for students. The preceding presented plausible positioning for the student to be the front of the classroom, aiding them to focus and understand that once in this location, expectations are required for learning. The position facilitates fewer visual and tactile aberrations which can cause students with ASD to become overwhelmed within an environment. However, ultimately the student and their mentor would determine the best location for the student positioning within the classroom. Hence the benefit of them being involved in the design process. Each individual with ASD responds differently to certain scenarios, where some individuals like to have sufficient space and other individuals prefer to be situated closer to assistants or teachers. Suggested layouts generated as part of this workflow could help ascertain a student's ideal location on a case-by-case basis, thus simplifying the process. The layouts provide a suggestive method for space layouts within the classroom while allocating specific or ideal locations for students with ASD or other SpLDs. Once the design layout was confirmed, Step 4 involved importing the layout into a communication platform. In this study the application analysis presented Dalux as the most suitable current construction tool with AR capabilities via its TwinBIM plugin. When uploaded, the model can be downloaded onto any mobile/tablet device with AR Core, via a Dalux BIM Viewer application. In this study a Samsung Galaxy Tab S3 was used for downloading the model data onto the viewer to showcase the newly established layouts. The design layout of the space was aligned through the application to allow the digital layout to overlay with the real-time studio (Figure 3). 


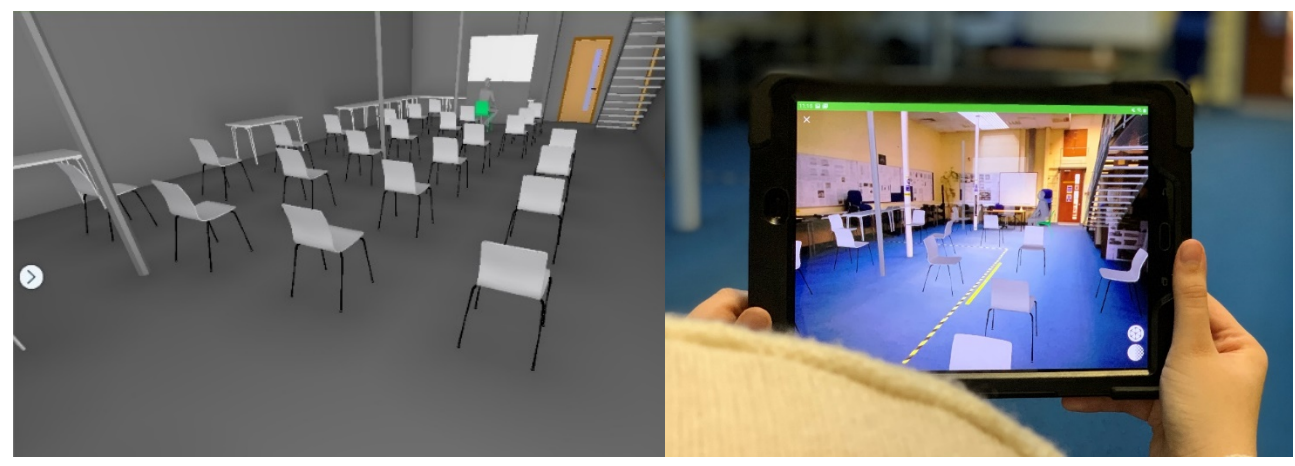

Figure 3. Design layout displayed using both virtual and augmented reality.

The proposed workflow resulted in hypothetical layouts being developed that could aid space layout design and communication. This method can allow designers and users to analyse and define the necessary links to evaluate solutions for the specified space, determining functionality and whether proposals are feasible. The output obtained from this workflow elevates its potential in aiding space layout planning, suggesting that the AR views could be exhibited to help those who need assistance in understanding the newly formed layout.

\section{Discussion}

This paper has shown the potential for a technology enabled workflow to have the ability to provide elevated assistance in the space planning and communication process. There is potential for resources such as Dynamo scripting, or the generative design tool available in Revit, to enable the generation of a variety of layouts, potentially determining the optimum layout to suit the circumstances required which can then be produced through AR. Incorporating visual methods can assist the designer in reconfiguring and understanding a space. Alongside supporting designers, the design platform and AR output provides a visual aid for students on the autism spectrum, benefiting their needs and stimulating their confidence in transitioning to new teaching environments. AR provides them with an exact visual representation of the room from their perspective, promoting familiarity within their classroom environment. The use of technology can provide a comfortable environment, thus promoting their educational experience. Looking forward, the integration of pre-recorded AR sessions, catered to the students, could provide the individuals with the opportunity to review the layout and process the changes over a period of time. These images could be incorporated into a social story (a personalised short story depicting a social situation that may be faced by the individual affected by ASD). Using this tool gives the ASD learner time to process the information prior to entering the social situation. It is however acknowledged that this is a first stage scoping study, with more work required to develop the overall concept and refine 
the workflow. Future work could explore the opportunities afforded by generative design and trial additional AR and VR products to determine the optimum solution.

\section{Acknowledgement}

This work has been developed from an undergraduate student research paper. The authors would like to thank Leica Geosystems for making the laser scan and initial model available for use. We would also like to thank Dalux for their assistance in the use of their platform.

\section{References}

Ciolfi, L. (2004). Understanding spaces as places: extending interaction design paradigms. Cognition, Technology \& Work, 6, 37-40. doi: 10.1007/s10111-003-0139-6.

Comiskey, D., Alexander, G., Hazlett, D., McCartan, K. and O'Boyle, L. (2016). The influence of space layout, technology and teaching approach on student learning. International Congress for Architectural Technology. Retrieved from: http://rua.ua.es/dspace/handle/10045/55246

Ganz, B. J., (2007). Classroom Structuring Methods and Strategies for Children and Youth with Autism Spectrum Disorders. Exceptionality, 15(4), 249-260. doi:10.1080/09362830701655816.

Guo, Z. and Li, B. (2017). Evolutionary approach for spatial architecture layout design enhanced by an agent-based topology finding system. Frontiers of Architectural Research, 6(1), 53-62. doi: 10.1016/j.foar.2016.11.003.

Holtz, D. K., Ziegert, K. A., Baker, D. C., Maloney, V. M., Snow, C. S. (2004). Life Journey through Autism : An Educator's Guide to Autism. United States: Dayna International and Organisation for Autism Research.

Jansen, D., Petry, J., Ceulemans, E., Noens, I., Baeyens, D. (2017). Functioning and participation problems of students with ASD in higher education : which reasonable accommodations are effective. European Journal of Special Needs Education, 32, 71-81. doi: 10.1080/08856257.2016.1254962.

Lui, R., Salisbury, J., Vahabzadeh, A. and Sahin, N. (2017). Feasibility of an Autism-Focused Augmented Reality Smartglasses System for Social Communication and Behavioral Coaching. Frontiers in Pediatrics, 5, 145. doi: 10.3389/fped.2017.00145.

NHS. (2019). What is Autism. Retrieved November 7, 2020, from https://www.nhs.uk/

Oblinger, D. (2005). Leading the Transition from Classrooms to Learning Spaces. EDUCAUSE Quarterly, 28, 14-18. Retrieved from: https://er.educause.edu/

Robson, C. and McCartan, K. (2016). Real World Research. $4^{\text {th }}$ Ed. Chichester: Wiley

Rodgers, H. and McCluney, J. (2020). Prevalence of Autism (including Asperger Syndrome) in School Age Children in Northern Ireland Annual Report 2020. Retrieved from Northern Ireland, Department of Health Web site: https://www.health-ni.gov.uk/

Sargent, J., 2019. VR and AR: Visualising the future of construction. Retrieved from KHL Group. Retrieved from https://www.khl.com/ 
Scott, I. (2009). Designing learning spaces for children on the autism spectrum. Good Autism Practice (GAP). 10(1), 36-51.

Stackhouse, J. (2018). The impact of 'place' on autistic children. Retrieved from: https://tinyurl.com/18f9g0q6

Valencia, K., Rusu, C., Quinones, D. and Jamet, E. (2019). The Impact of Technology on People with Autism Spectrum Disorder: A Systematic Literature Review. Sensors 19, 20. Doi: $10.3390 / \mathrm{s} 19204485$

Yin, R. K. (2018). Case study research and applications : design and methods. Los Angeles: Sage Publications Inc.

Zeppetella, K. (2009). Ten Effective Practices for Students with Asperger's Syndrome. St John's Fishers College. Education Masters. Retrieved from https://www.sjfc.edu/ 